\title{
Early Hepatic Encephalopathy after a Transjugular Intrahepatic Portosystemic Shunt Procedure: Case Report
}

\author{
Miriam Viviane Baron 1*, Gabriela Di Lorenzo Garcia Scherer², Michele Paula dos Santos², \\ Andres Felipe Mantilla Santamaria ${ }^{3}$, Cristine Brandenburg ${ }^{4}$, Isabel Cristina Reinheimer ${ }^{1}$, \\ Marcus Vinicius de Mello Pinto5, Aline Ronis Sampaio5, Alexandre Gomes Sancho6, \\ Esteban Fortuny Pacheco ${ }^{7}$, Felice Picariello ${ }^{8}$, Danielle de Mello Florentino ${ }^{9}$, Joice Nedel Ott ${ }^{1}$, \\ Carolina Gonçalves Pinheiro'0, Nathália Ken Pereira Iketani11, Sonia Carvalho' ${ }^{12}$, \\ Patrícia Froes Meyer13, Bartira Ercília Pinheiro da Costa1,2 \\ ${ }^{1}$ Postgraduate Program in Medicine and Health Sciences of the Pontifical Catholic University of Rio Grande do Sul (PUC/RS), \\ Porto Alegre, Rio Grande do Sul, Brazil \\ ${ }^{2}$ School of Medicine of the Pontifical Catholic University of Rio Grande do Sul (PUC/RS), Porto Alegre, Rio Grande do Sul, Brazil \\ ${ }^{3}$ Industrial University of Santander (UIS), Bucaramanga, Colombia \\ ${ }^{4}$ University of Vale do Acaraú (IDJ/UVA), Ceará, Brazil \\ ${ }^{5}$ Celulare Institute, Rio de Janeiro, Brazil \\ ${ }^{6}$ Unigranrio, Rio de Janeiro, Brazil \\ ${ }^{7}$ Universidad Finis Terrae, Santiago, Chile \\ ${ }^{8}$ Università degli Studi di Napoli Federico II, Napoli, Italy \\ ${ }^{9}$ Uniredentor University, Rio de Janeiro, Brazil \\ ${ }^{10}$ Postgraduate Program in Health Sciences of the Faculty of Medicine of ABC (FABC), São Paulo, Brazil \\ ${ }^{11}$ Hospital Beneficiente Portuguesa of Belém, Pará, Brazil \\ ${ }^{12}$ Rigshospital, Copenhagen East, København, Denmark \\ ${ }^{13}$ Centro Universitário do Rio Grande do Norte, Natal, Brazil \\ Email: *miriambaron9@gmail.com
}

How to cite this paper: Baron, M.V. Scherer, G.D.L.G., dos Santos, M.P., Santamaria, A.F.M., Brandenburg, C., Reinheimer, I.C., de Mello Pinto, M.V., Sampaio, A.R., Sancho, A.G., Pacheco, E.F., Picariello, F., de Mello Florentino, D., Ott, J.N., Pinheiro, C.G., Iketani, N.K.P., Carvalho, S., Meyer, P.F. and da Costa, B.E.P. (2021) Early Hepatic Encephalopathy after a Transjugular Intrahepatic Portosystemic Shunt Procedure: Case Report. Open Journal of Gastroenterology, 11, 81-88. https://doi.org/10.4236/ojgas.2021.116009

Received: May 26, 2021

Accepted: June 26, 2021

Published: June 29, 2021

\begin{abstract}
Patients with decompensated hepatic cirrhosis may present systemic alterations and dysfunction of multiple organs. Ascites, portal hypertension, esophageal varices, and hepatic encephalopathy are common complications arising from cirrhosis. The aim of this paper is to report a case of a patient with liver cirrhosis and the complications of a transjugular intrahepatic portosystemic shunt. Male, elderly, and ex-alcoholic, diagnosed with liver cirrhosis, ascites, and esophageal varices. He underwent transjugular intrahepatic portosystemic shunt due to portal hypertension and returned to the hospital after 24 hours with agitation and mental confusion. He had a bowel movement stop, neurological worsening, loss of renal function, hepatic hydrothorax, hepatic encephalopathy, hypernatremia, hypocalcemia, and hy-
\end{abstract}


Copyright @ 2021 by author(s) and Scientific Research Publishing Inc. This work is licensed under the Creative Commons Attribution International License (CC BY 4.0).

http://creativecommons.org/licenses/by/4.0/ pophosphatemia. He underwent a new procedure to occlude the transjugular intrahepatic portosystemic shunt, showing improvement of the mental status and ascites. However, continued with decompensation and hydro-electrolytic disorders. He evolved with worsening of the ventilatory pattern, and neurological and renal function, with a fatal outcome. Esophageal varices due to portal hypertension can be corrected with the transjugular intrahepatic portosystemic shunt. However, complications such as hypernatremia, hepatic hydrothorax, and hepatic encephalopathy may occur. Therefore, there is a need for reintervention to shunt or reduce its caliber. Thus, for patients with advanced age and decompensated cirrhosis, the potential risks and benefits of this procedure should be carefully evaluated due to the risk of complications and death.

\section{Keywords}

Ascites, Hypernatremia, Portasystemic Shunt Transjugular Intrahepatic, Hepatic Encephalopathy

\section{Introduction}

Liver cirrhosis is a disease in which normal liver tissue is replaced by fibrous tissue [1]. Only one-third of people with cirrhosis are aware of their condition. $\mathrm{Pa}$ tients usually remain asymptomatic until a decompensation occurs, and further clinical and radiological evaluation be performed [2].

When symptoms appear, cirrhosis may already be decompensated, which means hemodynamic and immune changes, with potential multiple organ dysfunction [3] [4]. Common complications from cirrhosis are ascites, portal hypertension, esophageal varices (EV), and hepatic encephalopathy (HE) [5]. The management of the disease includes close monitoring, and, for more advance disease or patients with complications, the referral to a transplant center [2].

The EV resulting from portal hypertension can be corrected with the procedure of transjugular intrahepatic portosystemic shunt (TIPS) [6]. This procedure creates an artificial passage, allowing the blood to pass directly from the portal system to the systemic circulation, decreasing the pressure in the portal system which is elevated due to liver cirrhosis [7]. However, this procedure can cause ammonia to pass into the system, being able to reach the brain, and developing Post-TIPS HE [7], which is a complication that occurs in $25 \%-33 \%$ of patients undergoing TIPS and is usually resolved with protein restriction and the use of lactulose or neomycin sulfate. However, in about 5\% - 10\% of patients, HE needs to be solved with a new intervention to occlude the TIPS or reduce its diameter [8].

The main complication of TIPS is its occlusion, which can lead to secondary complications such as new EV bleeding and death [9]. We present the report 
of a patient who had HE after TIPS, requiring TIPS occlusion. However, the patient had other diseases due to decompensated cirrhosis. The atypical presentation, torpid progression, unresponsiveness, and ominous outcome are important elements worth spreading with the objective of construct new collaborative knowledge.

\section{Case Report}

Male patient, white, 74 years old. Former smoker and drinker with 15 years of abstinence. Diagnostic of stage 4 liver cirrhosis complicated with ascites, and within the waitlist for liver transplantation. History of upper gastrointestinal bleeding with severe hemodynamic instability and presence of sclerotic EV. Comorbid diseases such as hypothyroidism and microcytic anemia. Managed at home with furosemide, spironolactone, omeprazole, levothyroxine, lactulose, and propranolol.

The patient was brought to the emergency room in January 2020 due to mental confusion and agitation, 24 hours after being discharged from a TIPS procedure. He was hemodynamically stable, with a Glasgow coma scale score of 10 and an auscultation with crackles in the right lung. The abdomen showed signs of ascites, with discomfort on palpation. The extremities were well-perfused without edema. The chest $\mathrm{x}$-ray showed hepatic hydrothorax $(\mathrm{HH})$. No structural changes in the brain were observed in the CT scan. A Child-Pugh class A classification was established.

On the next day, the patient was confused and agitated, with signs of dehydration, and a reduction of the ascites volume. The blood count showed thrombocytopenia and lymphopenia. Progressive neurological worsening was observed, and severe hypokalemia found, requiring diet by enteral tube, and transferred to the intensive care unit where invasive mechanical ventilation was inittiated because of hypoxemic ventilatory failure. An ultrasound examination at the bedside showed pleural effusion (PE) in the right lung,

Since the surgery, the patient had not defecated. An abdominal CT scan (Figure 1) reported a liver with chronic liver disease, presence of the porto-cava TIPS; portal hypertension, EV near the gastric fundus, and increase diameter of the portal vein. Angiotomography of the abdominal aorta (Figure 2) did not reveal significant occlusion.

Acute kidney injury was noticed, with a secondary metabolic acidosis. The patient evolved with hypernatremia and hypocalcemia secondary to repeated enemas. Stent implantation in the right hepatic vein without a complete opening was performed, intending to decrease the blood flow from the portal system to the systemic circulation looking forward to the TIPS occlusion, as an attempt to solve HE. A fracture of the stent occurred and the hydrophilic thread was buried in the right jugular vein.

Postoperative improvement was observed, Glasgow coma scale score went up to 14 , and the patient was extubated. Then hypokalemia, hypernatremia, 




Figure 1. CT scan of the abdomen. Liver with attenuation coefficient within normal limits, reduced dimensions, blunt edges, irregular contours, and enlarged caudate lobe. Orange arrow: Blunt edges. Red arrow: Enlarged caudate lobe.

hypophosphatemia, worsening renal function, and anemia were noticed. The patient evolved with bradycardia, hypotension, diarrhea, hypoperfusion, worsening of the neurological and ventilatory pattern, requiring reintubation.

A progressive neurological impairment was established despite the hydro-electrolytic correction; with persisting edema in the upper and lower limbs, hypotension, oliguria, hypothermia, vomiting and liquid stools. Finally, the patient developed a presumed ischemic stroke, dying as a result of a neurogenic shock.

\section{Discussion}

This case shows an ex-alcoholic patient in abstinence, with decompensated liver cirrhosis, acute kidney injury, hypernatremia, ascites, $\mathrm{HH}$, portal hypertension as a result of the cirrhosis, which resulted in EV and a TIPS procedure for 


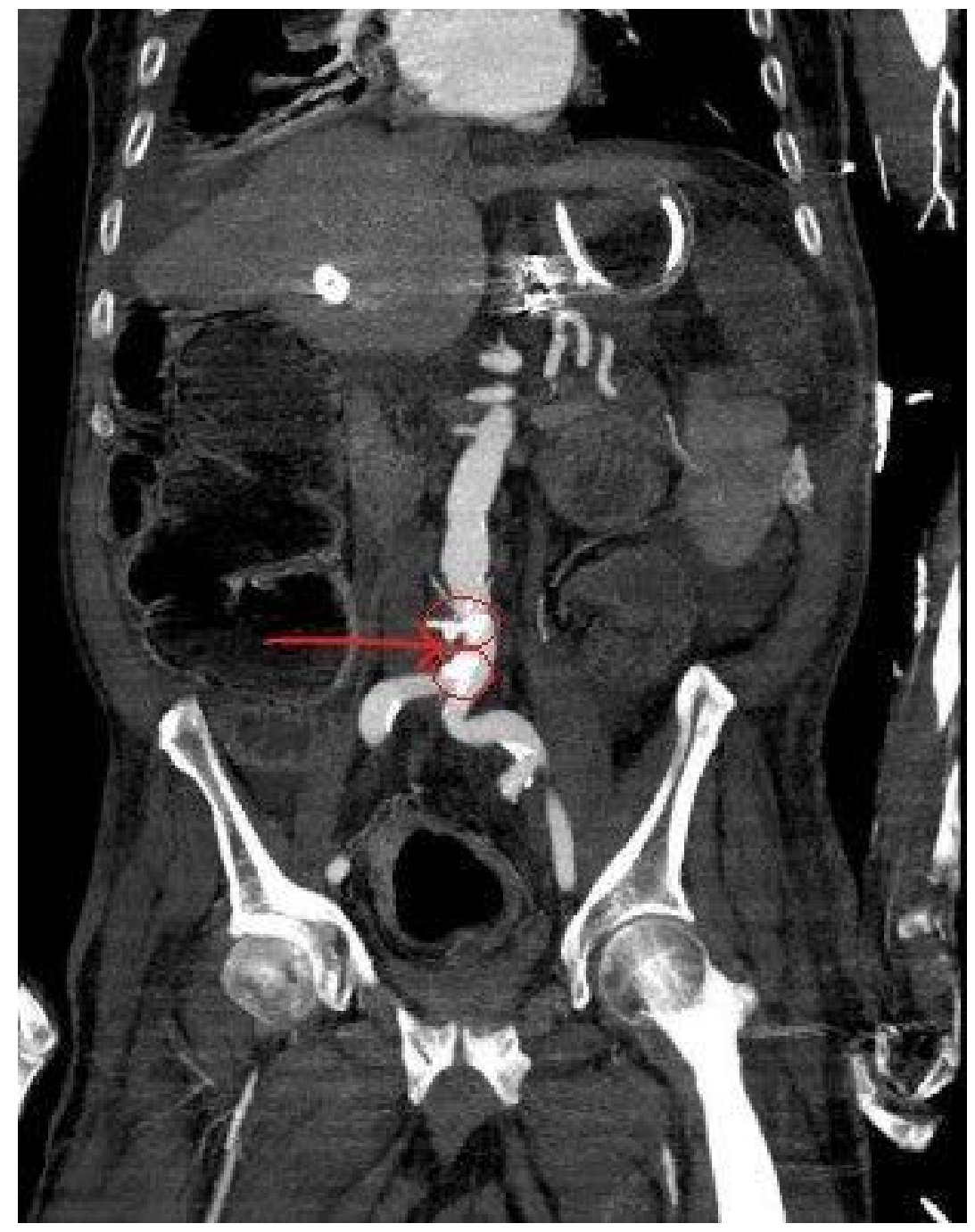

Figure 2. Angiotomography of the abdominal aorta. Patent abdominal aorta, with diffuse parietal irregularities, including calcified plaques and soft tissue density, without significant stenosis. Common right internal and external iliac arteries, with predominantly calcified plaques, with slight luminal reduction. Red arrow: Calcification of the abdominal aorta.

its correction. After the procedure, the patient developed HE, a problem that occurs in only $12 \%$ of patients who undergo TIPS, and that commonly develops 24 months after the procedure [10].

$\mathrm{HE}$ is a severe neuropsychiatric complication of cirrhosis and portal-systemic deviation, with a wide variety of clinical symptoms, ranging from subtle impairment of mental status to coma [11] [12]. After decompensated, cirrhosis becomes a systemic disease, with dysfunction of multiple organs and systems, including the immune system-leaving patients highly susceptible to bacterial infections [4]. The decompensation of cirrhosis represents a no return point in the prognosis since the average survival falls from 12 years in compensated cirrhosis to two years in decompensated [4]. The main complications of cirrhosis are bleeding from EV, HE, and ascites [3], whereas the main complications of the 
decompensated cirrhosis are the effects caused by reduced effective blood volume, [4] evoking compensatory responses that activate vasoconstrictors and sodium and water retention factors, such as the renin-angiotensin-aldosterone system and the sympathetic nervous system [3] [13].

Some complications developed by the patient were HH and PE; the PE commonly develops in patients with cirrhosis and portal hypertension in the absence of cardiopulmonary disease; although the development of $\mathrm{HH}$ remains unclear, the most accepted explanation is that $\mathrm{HH}$ results from a direct passage of ascitic fluid into the pleural cavity through a defect in the diaphragm-due to high abdominal pressure and negative pressure within the pleural cavity [14].

The patient was diagnosed with EV; which is one of the main complications of cirrhosis [4], their frequency varies from $30 \%$ to $40 \%$ in compensated cirrhosis and $60 \%$ in patients with ascites. EV is directly related to portal hypertension, which caused increased portal resistance, developing collateral circulation and varices [15]. Due to portal hypertension, the patient underwent the TIPS [6], in which the shunt reduces the resistance of the portal vein, diminishing portal hypertension in patients with cirrhosis and relieving ascites [10]. HE was developed close after the TIPS, requiring a new hospitalization and stent implantation to occlude the TIPS.

Even after the TIPS occlusion and a brief improvement of the sensorium, it is believed that one of the most important factors for the patient's death was the accentuated hydro-electrolytic disorder due to decompensated cirrhosis, especially the severe hypernatremia. An epidemiological study has shown that severe dysnatremias are not common in cirrhotic patients, but hyponatremia is the main hydro-electrolytic imbalance associated with cirrhosis, with a prevalence of $50 \%$ in patients with ascites [3]. However, hypernatremia is a rare condition associated with an adverse prognosis, caused by the loss of hypotonic fluids due to glycosuria and/or lactulose-induced diarrhea [3], as occurred in this case due to the administration of lactulose and prokinetics.

An interruption of hepatic metabolism of ammonia and other neurotoxins due to TIPS is common [9]. However, there was an unusual evolution in the present case, such as the development of hypernatremia, $\mathrm{HH}$, and $\mathrm{HE}$ just one week after the TIPS. Despite being indicated for the treatment of EV, the literature shows a relatively high mortality rate after TIPS due to poor selection of patients for the procedure [9]. In addition, advanced age at the time of TIPS placement is a negative predictor of survival [9].

Therefore, it is believed that the ominous evolution was possibly related to decompensated liver cirrhosis associated with severe and difficult to control hypernatremia, which brought refractoriness to the therapeutic measures instituted. Patients with decompensated liver cirrhosis may have hemodynamic changes and multiple organ dysfunction. EV due to portal hypertension can be corrected with the TIPS. However, this procedure can result in unusual complications, such as hypernatremia, $\mathrm{HH}$, and HE. Therefore, there is a need for rein- 
tervention to occlude the TIPS or to reduce its caliber to improve the clinical condition. Thus, in patients with advanced age and decompensated cirrhosis, potential risks and benefits of the TIPS should be evaluated to the detriment of other treatments due to the risk of complications and death.

\section{Acknowledgements}

This study was funded in part by the Coordination of Improvement of Higher Level Personnel-Brazil (CAPES) - Finance Code 001, by the Foundation for Research Support of the State of Rio Grande do Sul (FAPERGS) and by the Scholarship/Research Program for Undergraduate Students (BPA/PUCRS).

\section{Conflicts of Interest}

The authors declare no conflicts of interest regarding the publication of this paper.

\section{References}

[1] Zhou, W.-C., Zhang, Q.-B. and Qiao, L. (2014) Pathogenesis of Liver Cirrhosis. World Journal of Gastroenterology, 20, 7312-7324. https://doi.org/10.3748/wjg.v20.i23.7312

[2] Smith, A., Baumgartner, K. and Bositis, C. (2019) Cirrhosis: Diagnosis and Management. American Family Physician, 100, 759-770. https://www.aafp.org/afp/2019/1215/p759.html

[3] Bernardi, M. and Zaccherini, G. (2018) Approach and Management of Dysnatremias in Cirrhosis. Hepatology International, 12, 487-499.

https://doi.org/10.1007/s12072-018-9894-6

[4] European Association for the Study of the Liver (2018) EASL Clinical Practice Guidelines for the Management of Patients with Decompensated Cirrhosis. Journal of Hepatology, 69, 406-460. https://doi.org/10.1016/j.jhep.2018.03.024

[5] Almani, S.A., Memon, A.S., Memon, A.I., Shah, M.I., Rahpoto, M.Q. and Solangi, R. (2008) Cirrhosis of Liver: Etiological Factors, Complications and Prognosis. Journal of Liaquat University of Medical and Health Sciences, 7, 61-66. https://doi.org/10.22442/jlumhs.08720145 https://www.lumhs.edu.pk/jlumhs/Vol07No02/pdfs/v7n2oa01.pdf

[6] Buechler, C., Haberl, E.M, Rein-Fischboeck, L. and Aslanidis, C. (2017) Adipokines in Liver Cirrhosis. International Journal of Molecular Sciences, 18, Article No. 1392. https://doi.org/10.3390/ijms18071392

[7] Rajesh, S., George, T., Philips, C.A., Ahamed, R., Kumbar, S., Mohan, N., et al. (2020) Transjugular Intrahepatic Portosystemic Shunt in Cirrhosis: An Exhaustive Critical Update. World Journal of Gastroenterology, 26, 5561-5596. https://doi.org/10.3748/wjg.v26.i37.5561

[8] Carreiro, G., Moreira, A., Murad, F.F., Azevedo, F. and Coelho, H.S.M. (2001) TIPS-Anastomose portossistêmica intra-hepática transjugular. Revisão. Arquivos de Gastroenterologia, 38, 69-80. https://doi.org/10.1590/S0004-28032001000100013

[9] Strunk, H. and Marinova, M. (2018) Transjugular Intrahepatic Portosystemic Shunt (TIPS): Pathophysiologic Basics, Actual Indications and Results with Review of the Literature. Rofo, 190, 701-711. https://doi.org/10.1055/a-0628-7347

[10] Chen, Y., Qiu, H. and Zhang, X. (2019) Transjugular Intrahepatic Portal Shunt in 
the Treatment of Portal Hypertension Due to Cirrhosis: Single Center Experience. BMC Surgery, 19, Article No. 191. https://doi.org/10.1186/s12893-019-0659-5

[11] Hadjihambi, A., Arias, N., Sheikh, M. and Jalan, R. (2018) Hepatic Encephalopathy: A Critical Current Review. Hepatology International, 12, 135-147. https://doi.org/10.1007/s12072-017-9812-3

[12] Amodio, P. (2018) Hepatic Encephalopathy: Diagnosis and Management. Liver International, 38, 966-975. https://doi.org/10.1111/liv.13752

[13] Schrier, R.W., Arroyo, V., Bernardi, M., Epstein, M., Henriksen, J.H. and Rodés, J. (1988) Peripheral Arterial Vasodilation Hypothesis: A Proposal for the Initiation of Renal Sodium and Water Retention in Cirrhosis. Hepatology, 8, 1151-1157. https://doi.org/10.1002/hep.1840080532

[14] Lv, Y., Han, G. and Fan, D. (2018) Hepatic Hydrothorax. Annals of Hepatology, 17, 33-46. https://doi.org/10.5604/01.3001.0010.7533

[15] Maruyama, H., Kobayashi, K., Kiyono, S., Ogasawara, S., Ooka, Y., Suzuki, E., et al. (2019) Incidence and Hemodynamic Feature of Risky Esophageal Varices with Lower Hepatic Venous Pressure Gradient. International Journal of Medical Sciences, 16, 1614-1620. https://doi.org/10.7150/ijms.37040 